\title{
ПРИЕМНЫЕ УСТРОЙСТВА ДЛЯ ВОЛС: СПОСОБЫ ПРИЕМА И ОБРАБОТКИ СИГНАЛОВ РАЗЛИЧНЫХ ТИПОВ
}

\author{
Н.И. Варава, С. В. Пронин, М. Ю. Никоноров \\ "Оптоэлектронные технологии" (ООО «АИБИ»), info@optotech.ru, Санкт-Петербург
}

\begin{abstract}
При работе волоконно-оптических линий связи с сигналами определенной формы требуется согласование приемных и передающих модулей по динамическому диапазону оптических сигналов, рабочей длине волны, типам волокна и оптических разъемов. Чтобы удовлетворить столь многообразные требования к вОЛС для эксплуатации в условиях климатических, механических и электромагнитных воздействий разработаны комплекты функционально законченных модулей. Представлены оптические приемные для различных применений, в том числе - для систем управления и запуска устройств, коммутирующих значительные мощности.
\end{abstract}

\section{ВВЕДЕНИЕ}

Принципы работы фотонных средств на базе волоконно-оптических линий связи (ВОЛС) основаны на разных физических эффектах. Однако все системы ВОЛС обязательно имеют на одном конце линии лазер для передачи данных («1" или «0»), а на другом - фотоприемник для детектирования величины фототока. Обработку оптических сигналов, поступающих из ВОЛС, отличают некоторые особенности, связанные с характером сигнала. Однако внешние помехи (механические, климатические, электромагнитные) оказывают негативное воздействие на приемопередающий тракт, снижая отношение сигнал/шум. И наиболее уязвимым звеном в цепи устройств этой системы ВОЛС является фотоприемное устройство [1].

Проявление подобных качеств особенно заметно в условиях воздействия электромагнитных помех на приемопередающий тракт. В первую очередь причина их появления связана с необходимостью приема достаточно слабых сигналов. Другая причина кроется в специфике последующей обработки сигналов, которые отличаются произвольным характером передаваемой цифровой последовательности (сигналы имеют различную длительность и формат). Такой характер (т. е. неограниченный формат передачи) вызывает необходимость поддерживать работу приемника в режиме максимальной чувствительности. Дело в том, что работу разнообразных систем автоматического регулирования усиления (АРУ) по непрерывным сигналам характеризует некоторое время реакции. Именно поэтому невозможно обрабатывать сигналы, имеющие в своем спектре посто- янную составляющую. Решить задачу обработки входных сигналов, выраженных в цифровом виде и имеющих произвольный формат (в виде одиночных сигналов, в виде пакетных сигналов, в виде сигналов любой длительности и др.) достаточно проблематично, если требуется обеспечить максимальное быстродействие, высокую чувствительность одновременно с получением удовлетворительных выходных параметров. Да и часто оказывается, что не во всех ситуациях это необходимо. В то же время при создании конструкций приемных оптических модулей, предназначен ${ }^{-}$ ных для эксплуатации в условиях климатических, механических и электромагнитных воздействий, к их характеристикам надо проявлять особое внимание.

Специалисты различают несколько способов обработки сигналов произвольного формата [2] в приемниках для ВОЛС:

- с фиксированным уровнем определения логического состояния,

- с определением логического состояния по фронту и спаду входного импульса,

- с использованием инверсной кодировки входных сигналов,

- с использованием обработки сигналов близких к уравновешенным, т.е. без передачи постоянной составляющей.

Во всех приемниках, представленных в данной статье, аналоговая часть работает в линейном режиме (за исключением устройств, использующихся в оптронах). Динамический диапазон входных сигналов определяется в основном уровнем собственных шумов и напряжением питания. 


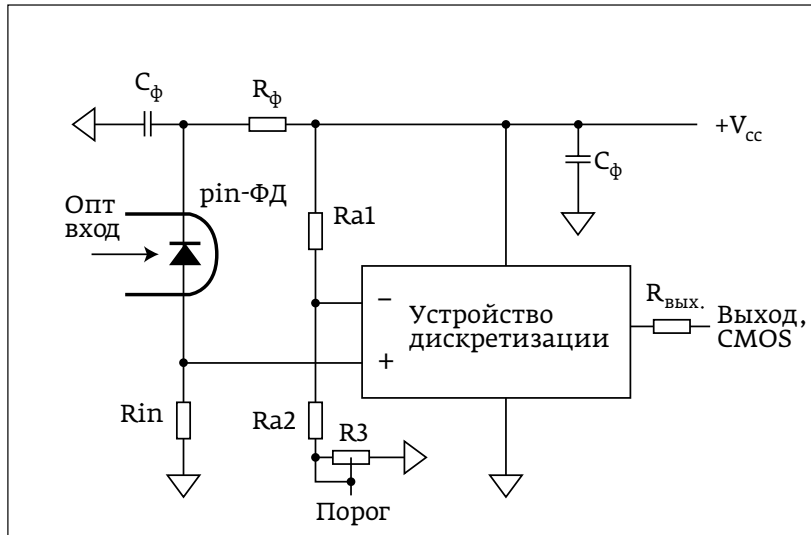

Puc. 1. Функциональная схема оптического приемного модуля OMRD-03-A/В с фиксированным уровнем определения согического состояния

\section{ОПТИЧЕСКИЕ ПРИЕМНЫЕ МОДУЛИ}

К приемным устройствам с фиксированным порогом определения логического состояния относятся оптические приемные модули OMRD-03-A/B, имеющие в своем составе линейную аналоговую часть и быстродействующее устройство дискретизации, а также OMRD-02-A/B и OMRS-01, OMRS-02, предназначенные для использования в составе волоконно-оптического оптрона (волстрона) [3]. Модули OMRD-03-A/B спроектированы с учетом возможности внешней регулировки порога срабатывания. Их выпускают для работы в двух спектральных диапазонах - 0,85 мкм (А) и 1,3-1,55 мкм (В). Тип волокна - SMF, MMF с диаметром световотовода $\leq 62,5 / 125$ мкм. OMRD-03-А снабжен оптическим разъемом типа розетка ST, OMRD-03-B - оптическим разъемом розетка FC, OMRD-03-B-pg - пигтейл с вилкой FC. Обе модификации конструктивно оформлены в герметичных металлических корпусах со штырьковыми выводами. К достоинствам данного типа модулей относится низкое потребление энергии по цепи питания. Это качество позволяет использовать их в системах синхронизации и передачи информации с датчиков, размещенных в труднодоступных местах и имеющих источник питания с ограниченным ресурсом. Функциональная схема изделий OMRD-03-A/B приведена на рис. 1.

Оптические приемные модули OMRD-02-A/B предназначены для использования в волоконнооптических оптронах и дистанционных выключателях. Они содержат в своем составе кроме ріпфотодиода еще усилитель фототока и выходной каскад, выполненный по схеме с обшим коллектором (OK). Указанный тип приемных модулей

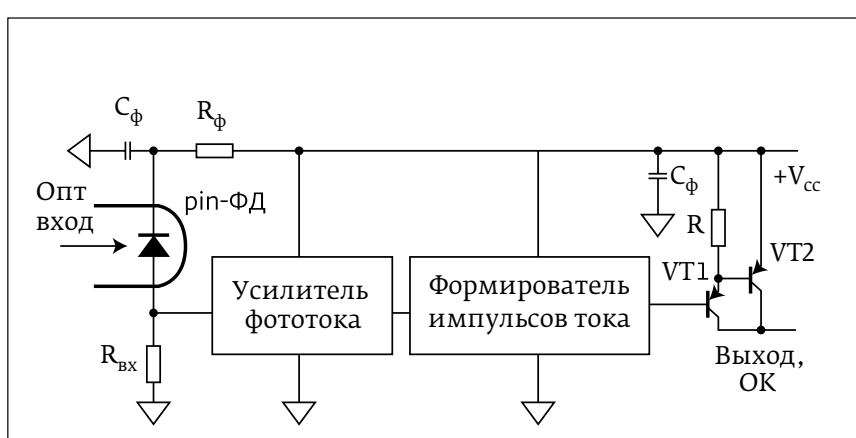

Puc. 2. Функциональная схема оптических приемников muna OMRS

отличает широкий диапазон питающих напряжений (3-12 В) и сниженное энергопотребление по цепи питания при отсутствии сигналов на входе. Модули выпускаются для двух оптических диапазонов с длинами волн 0,85 мкм (А) и 1,3-1,55 мкм (В). Они заключены в конструкцию из металлических герметичных корпусов, что обеспечивает защиту от внешних электромагнитных полей. Корпуса выполнены на стандартных основаниях типа 151.15-8 ПАЯ4.880.011-01 со штырьковыми выводами. Так как выходной каскад этих приемников работает в режиме насыщения, то их быстродействие оказывается не слишком высоким. Основное применение данных устройств - использование в устройствах передачи статусных сигналов (оптроны, оптореле и др.)

Специализированные оптические приемные модули OMRS-01 и OMRS-02 предназначены для применения в системах управления и запуска устройств, коммутирующих значительные мощности [3]. Это могут быть системы управления электродвигателями, электромагнитными ускорителями, разрядниками и др. Схема исполнения обоих модулей одинакова: фотодиод, усилитель фототока и выходной каскад с общим коллектором. Отличие состоит лишь в решении схемы выходного узла, позволяющего получить на выходе модуля импульсы тока в 2 А для OMRS-01 и 15 А для OMRS-02. Модули заключены в металлостеклянные герметичные корпуса из специального сплава с использованием стандартного основания типа 155.15-2 ПАЯ4.880.007-02, что очень важно при использовании их в условиях сильных электромагнитных воздействий. С этой же целью в модулях используется оптический разъем типа ST. Данный тип приемных модулей согласован по оптическому интерфейсу с передающим модулем OMTD-02 и предназначен для работы 


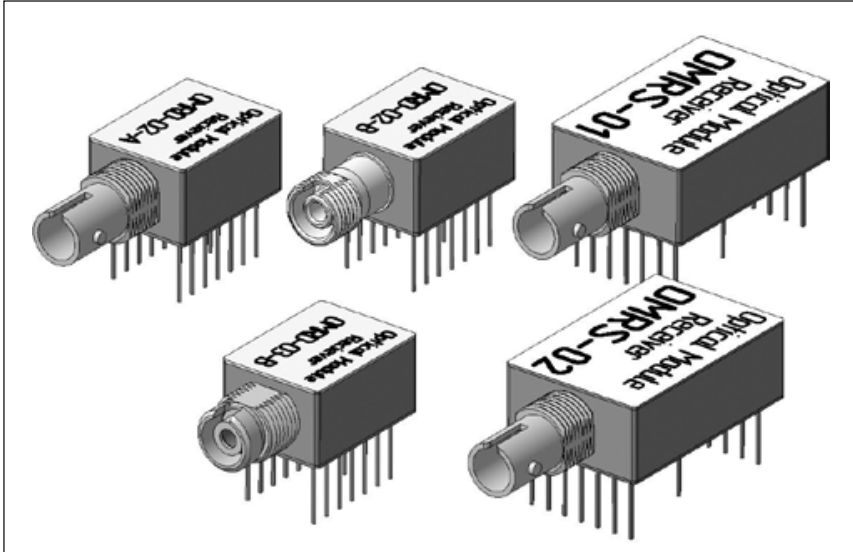

Puс. 3. Внешний вид приемных цифровых оптических модулей OMRD-02, OMRD-03, OMRS-01, OMRS-02

с многомодовым волокном (MMF) 62.5/125мкм. Приемные модули OMRS-01, OMRS-02 являются усовершенствованными аналогами разработан ${ }^{-}$ ных ранее ФПУ-Э и ФПУ-Э-м. Функциональная схема модуля данного типа приведена на рис. 2. Изделия OMRD-02, OMRD-03, OMRS-01, OMRS-02 составляют группу продукции по применению в основном для решения технологических и сервисных задач. Внешний вид этих модулей показан на рис. 3.

Следующая группа приемных оптических устройств включает в себя изделия OMRD-04 $(\lambda=0,85 \mathrm{MKM})$ и OMRD-01, OMRD-06 ( $\lambda=1,3-1,55 \mathrm{MKM})$. B них используется схема, определяющая логическое состояние по фронту и по спаду входного импульса. Данный тип схемных решений обеспечивает минимально достижимое значение уширения выходных сигналов по отношению к входным. Но такой результат требует расширения полосы аналоговой части приемника и отсутствия существенной дисперсии в ВОЛС. Для объектовых систем эти требования выполнимы, так как

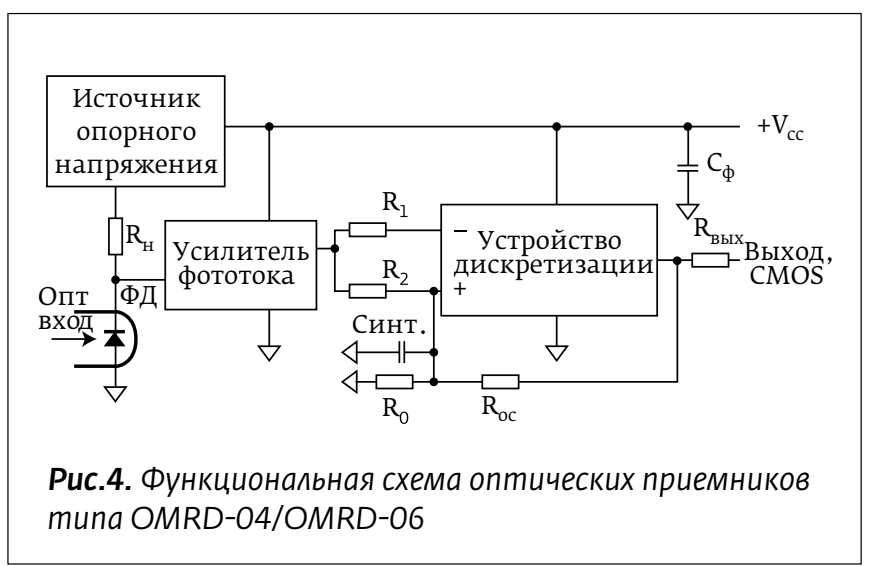

протяженность оптоволоконных линий относительно невелика, и полоса рабочих частот может быть увеличена за счет снижения чувствительности. В состав данного типа приемника входит pin-фотодиод, усилитель тока и высокочувствительный триггер Шмидта. Сигнал, поступающий с усилителя тока, дифференцируется, и короткие импульсы управляют работой триггера. Сам триггер выполнен на базе быстродействующего компаратора, охваченного положительной обратной связью. Оптический приемник OMRD-01 предназначен для работы с передатчиком OMTD-01-m, выполненным с использованием светодиода, и поэтому он в своем составе содержит дополнительный предварительный трансимпедансный усилитель. Функциональная схема оптических приемников OMRD-04 и OMRD-06 (рис. 4) и OMRD-01 (рис. 5). Данная группа приемных модулей предназначена для использования в системах передачи информации с произвольным форматом в сложных условиях эксплуатации. Тип оптического волокна MMF, SMF $\leq 62,5 / 125$ мкм. Конструктивно вышеуказанные модули выполнены в металлостеклянных корпусах на стандартном основании и оснащены оптическим разъеMom. OMRD-01, OMRD-06 имеют разъем типа FC, OMRD-04 - разъем типа ST. Разъем OMRD-06-pg выполнен в виде пигтейла, оконцованного вилкой FC. Внешний вид модулей данного типа представлен на рис. 6.

Для ВОЛС большой протяженности или с большим затуханием мы использовали в приемнике так называемую схему с инверсией обрабатываемых сигналов. В устройствах данного класса значение полосы рабочих частот минимизируется для получения максимального соотношения сиг-

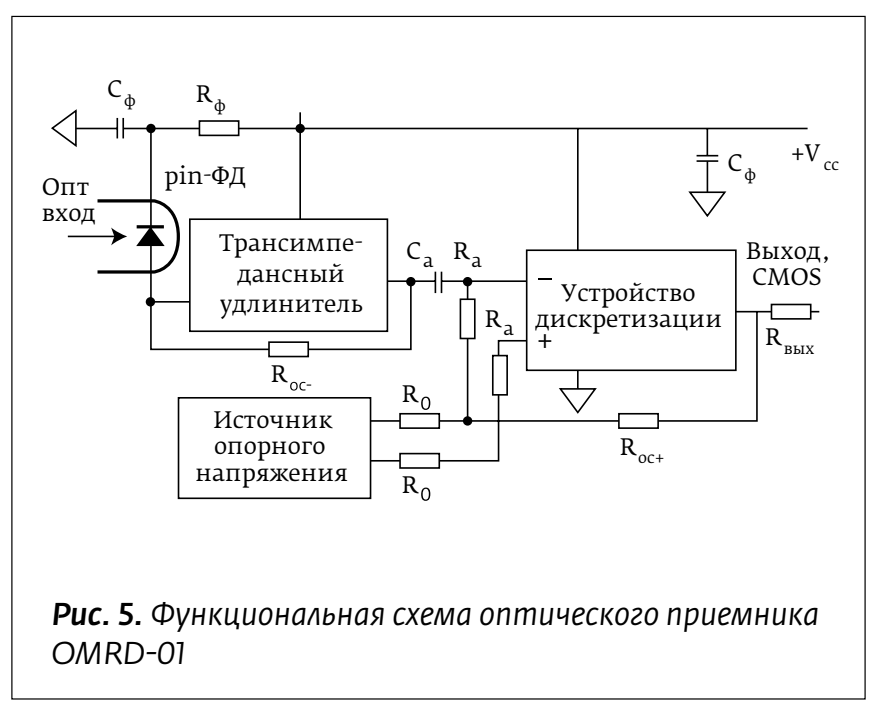




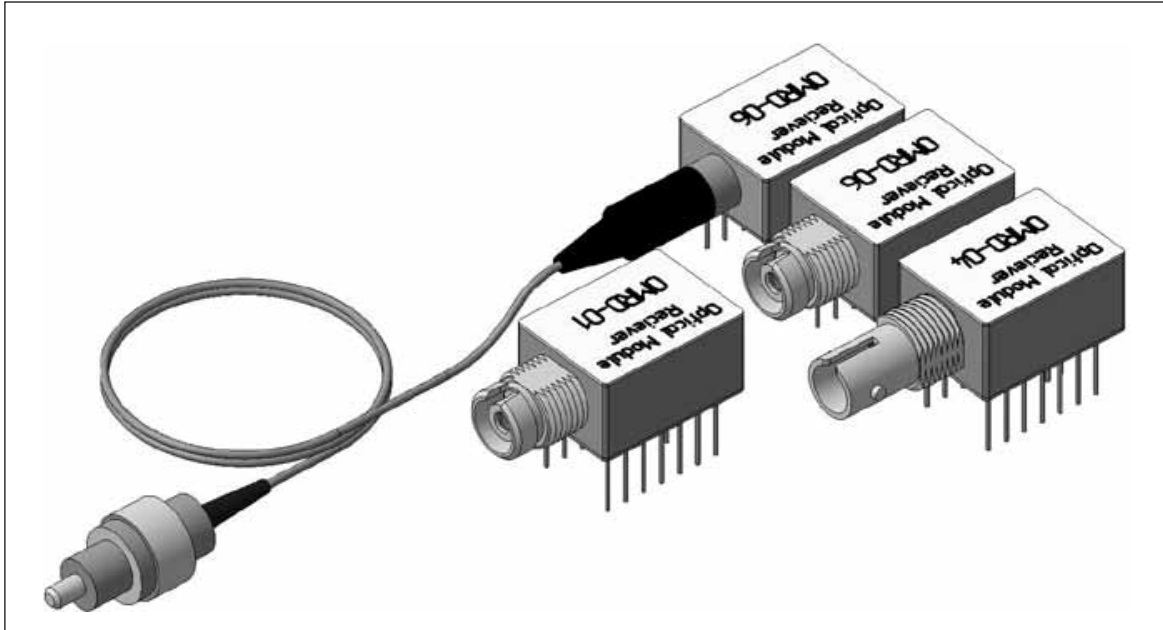

Puc. 6. Внешний вид приемных цифровых оптических модулей OMRD-01, OMRD-04, OMRD-06

ственной обработки одиночных и пакетных сигналов с максимально возможным (для каждого типа приемника она разная) соотношением сигнал/шум. Функциональная схема такого приемника OMRD-05 показана на рис. 7. Корпус изделия металлостеклянный с использованием стандартного основания типа 155.15-2 ПАЯ4.880.007-02. Оптический разъем - пигтейл, оконцованный вилкой типа FС. Приемник предназначен для работы в комплекте с передающим модулем OMTD-05, имеющим вход инверсии входного сигнала. Дополнительно в приемнике имеется устройство,

нал/шум на входе аналоговой части приемника. Отметим также, что в приемниках, использующих линейную часть динамического диапазона и обрабатывающих одиночные и пакетные сигналы, всегда существует проблема установки порога срабатывания устройства дискретизации. Причина кроется в отсутствии информации об амплитуде входного сигнала. Величина этого порога для получения минимального уширения импульсов на выходе в условиях ограниченной полосы частот должна быть равна половине амплитудного значения сигнала.

С этой целью была применена схема с использованием инверсной кодировки входных сигналов. Сигнал "1" (наличие излучения от оптического источника в волоконно-оптическом тракте соответствует "0" (отсутствие света) на входе оптического передатчика. В приемнике детектор сигнала определяет его амплитуду и автоматически устанавливает на входе устройства дискретизации величину порога, равную половине амплитуды. Уровень "0" в аналоговой части приемника определяется значением (для каждого типа приемника она разная) внутреннего источника опорного напряжения. На выходе цифровой части приемника кодировка сигнала соответствует кодировке на входе передатчика. Таким образом, достигается возможность каче- сигнализирующее о наличии на входе сигнала с амплитудой, достаточной для обработки его с заданным коэффициентом ошибки. Внешний вид модуля представлен на рис. 8.

И в заключение представляем тип приемного устройства, предназначенного для обработки сигналов, имеющих формат, близкий к уравновешенному (суммарная длительность "1" и “0» за некоторый характерный для реальной системы период равна друг другу), т.е. в спектре транслируемых сигналов практически отсутствует постоянная составляющая. Функциональная схема приемника OMRD-07-X, в котором реализуется вышеуказан ${ }^{-}$ ный принцип, представлена на рис. 9. Приемник выпускается для двух диапазонов скоростей 8 и 34 Мбит/с с рабочей длиной волны 1,3-1,6 мкм.

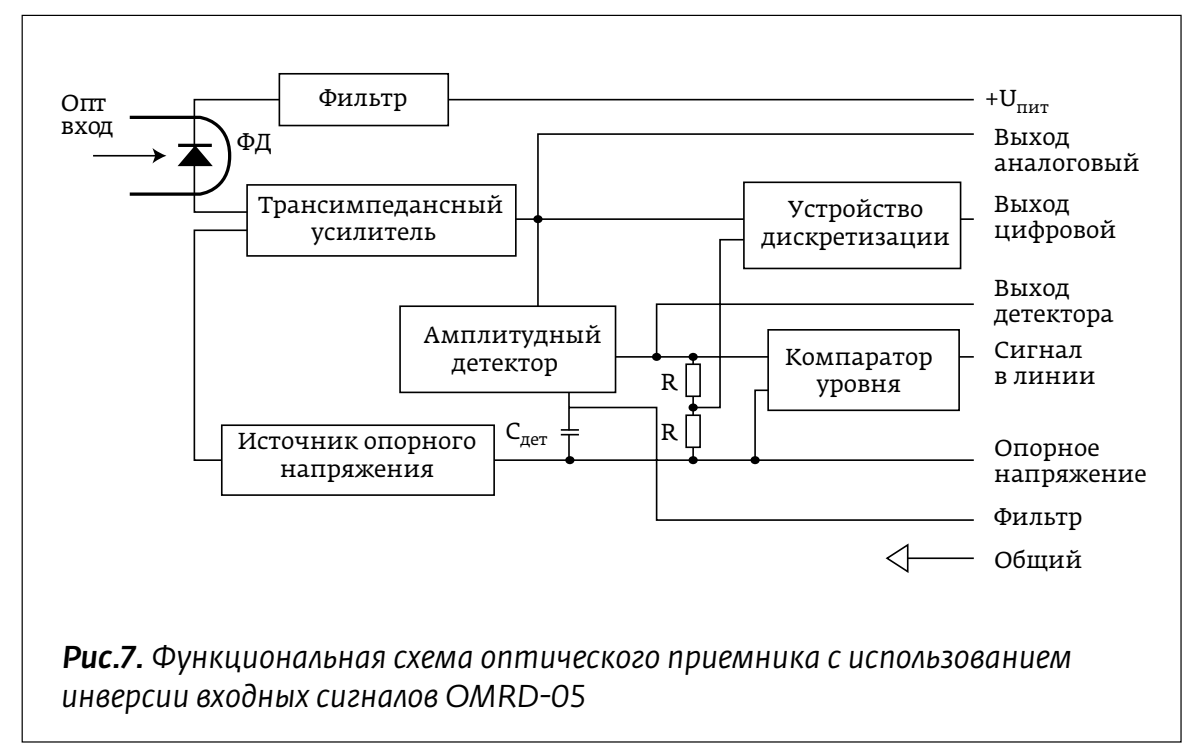




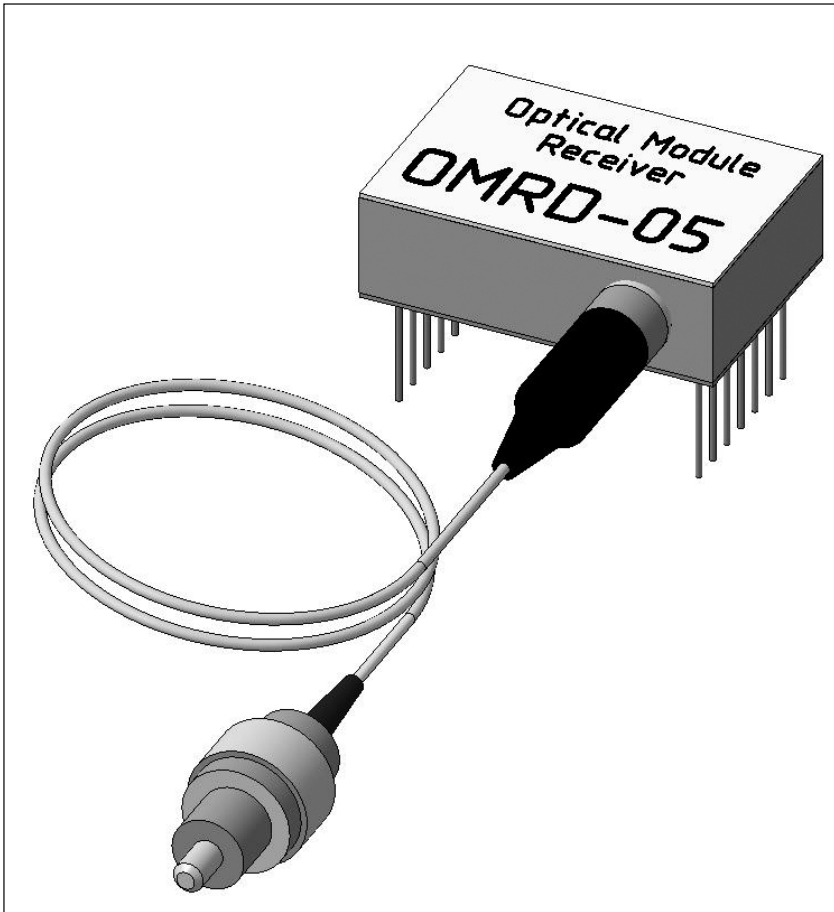

Puс.8. Внешний вид приемника цифровых оптических Сигналов OMRD-05

Модули конструктивно выполнены в металлостеклянных корпусах и снабжаются разъемами типа розетка $\mathrm{FC}$ или пигтейлом с вилкой $\mathrm{FC}$. Внешний вид оптических приемников OMRD-07-X представлен на рис. 10.

Отличительной особенностью приемных модулей OMRD-07-X является их способность (в зависимости от схемы включения) обрабатывать сигналы как неуравновешенные (одиночные, пакетные и др.), так и имеющие уравновешенный характер (равное количество «0» и "1»). Эта особенность может оказаться существенной, если приемник находится в зоне воздействия значительных электромагнитных полей. Приемники, обрабатывающие неуравновешен ${ }^{-}$ ные последовательности вне зависимости от соотношения сигнал/шум на их входе, всегда работают в режиме максимальной чувствительности. Перевод такого приемника в режим обработки уравновешенного сигнала существенно повышает его помехоустойчивость. Осуществляется это (см. рис. 9) подклю- чением внешнего конденсатора параллельно внутреннему $\mathrm{C}_{\text {диф. }}$ между усилителем фототока (УФТ) и входом устройства дискретизации (УД). Величина его определяет значение нижней рабочей частоты устройства. Отметим также, что пороговая чувствительность приемника при таком включении в два раза выше.

Оптические приемные модули OMRD-05, OMRD-07-X по применению также относятся к группе для использования в волоконно-оптических системах передачи информации.

\section{ЗАКЛЮЧЕНИЕ}

Появление целого ряда специализированных активных компонентов для объектовых и региональных ВОЛС вызвано условиями эксплуатации таких линий и достаточно широкому спектру решаемых с их помощью задач. Для поиска оптимального решения требуются, как правило, устройства, имеющие определенные характеристики как со стороны приемной части, так и передающей. Это относится в первую очередь к согласованию их по динамическому диапазону оптических сигналов, рабочей длине волны, типам волокна и оптических разъемов, а также единого конструктивного исполнения. Чтобы учесть столь многоликие требования устройств из этой области волоконной техники, мы разработали изделия в виде комплектов, состоящих из функционально законченных модулей: приемного модуля и передающего. При этом модули могут быть использованы и самостоятельно.

Также необходимо отметить, что в представленных разработках используются основополагающие оптические комплектующие, такие как лазерные диоды, фотодиоды, светодиоды и инте-

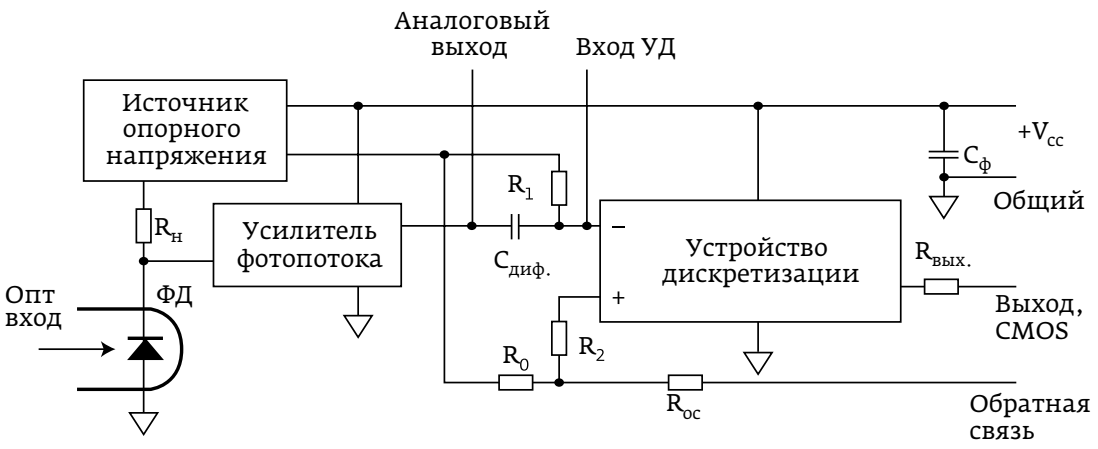

Puc.9. Функциональная схема оптического приемника OMRD-07-Х для обработки сигналов, имеющих уравновешенный формат 


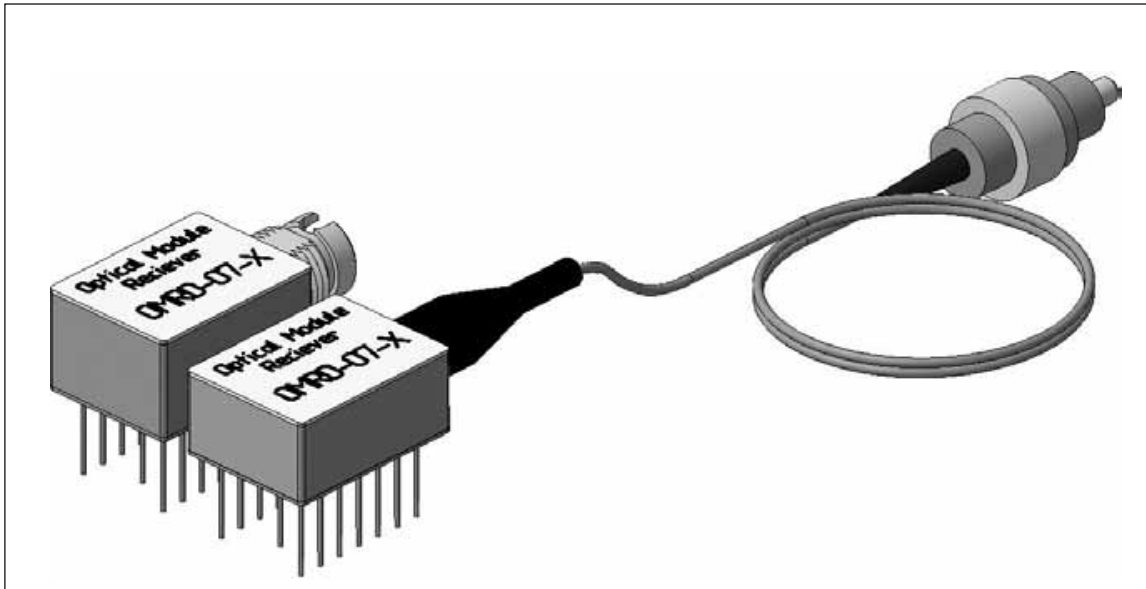

Pис.10 Внешний вид оптического приемника для обработки уравновешенных цифровых последовательностей в ВОЛС OMRD-07-X (в зависимости от производителя компонентов), по простоте реализации приемной части. Во многих случаях модули опережают по чувствительности и быстродействию изделия импортного производства, разработанные ранее для решения аналогичных задач.

K преимуществам разработанных изделий можно отнести также и конструкцию модулей, обеспечивающую стандартное подключение, высокую степень электромагнитной защищенности и стабильность эксплуатационных характеристик (в температурном диапазоне,

гральные схемы с самыми высокими значениями оптических и электрических параметров, достигнутыми на сегодняшний день. По этой причине представленные изделия имеют ряд преимуществ перед аналогичной продукцией отечественных производителей по отношению потребляемой мощности к выходной оптической мощности изменения влажности и пр).

\section{СПИСОК ЛИТЕРАТУРЫ}

1. Фриман Р. Волоконно-оптические системы связи. М.: Изд-во Техносфера, 2003. Friman R. Volokonno-opticheskie sistemy svyazi. M.: Izd-vo Tekhnosfera, 2003.

2. Winzer P. J., Essiambre R.-J. Advanced Optical Modulation Formats. Proce. of the IEEE. 2006;94 (5): 952-985.

3. URL: www.optotech.ru. 